\title{
Research on Quantitative Remote Sensing Monitoring Algorithm of Air Pollution Based on Artificial Intelligence
}

\author{
Yun Liu, ${ }^{1}$ Yuqin Jing, ${ }^{2}$ and Yinan $\mathrm{Lu} \mathbb{D}^{3}$ \\ ${ }^{1}$ School of Information Engineering, Chaohu University, Chaohu, China \\ ${ }^{2}$ School of Electronic Information Engineering, Chongqing Technology and Business Institute, Chongqing, China \\ ${ }^{3}$ School of Information Engineering, Nanchang University, Nanchang, China \\ Correspondence should be addressed to Yinan Lu; luyinan250@163.com
}

Received 1 November 2019; Accepted 30 November 2019; Published 4 March 2020

Guest Editor: Jia-Bao Liu

Copyright ( 92020 Yun Liu et al. This is an open access article distributed under the Creative Commons Attribution License, which permits unrestricted use, distribution, and reproduction in any medium, provided the original work is properly cited.

\begin{abstract}
When the current algorithm is used for quantitative remote sensing monitoring of air pollution, it takes a long time to monitor the air pollution data, and the obtained range coefficient is small. The error between the monitoring result and the actual result is large, and the monitoring efficiency is low, the monitoring range is small, and the monitoring accuracy rate is low. An artificial intelligence-based quantitative monitoring algorithm for air pollution is proposed. The basic theory of atmospheric radiation transmission is analyzed by atmospheric radiation transfer equation, Beer-Bouguer-Lambert law, parallel plane atmospheric radiation theory, atmospheric radiation transmission model, and electromagnetic radiation transmission model. Quantitative remote sensing monitoring of air pollution provides relevant information. The simultaneous equations are constructed on the basis of multiband satellite remote sensing data through pixel information, and the aerosol turbidity of the atmosphere is calculated by the equation decomposition of the pixel information. The quantitative remote sensing monitoring of air pollution is realized according to the calculated aerosol turbidity. The experimental results show that the proposed algorithm has high monitoring efficiency, wide monitoring range, and high monitoring accuracy.
\end{abstract}

\section{Introduction}

With the improvement of people's material living conditions, the advancement of science and technology, and the increased consumption of energy, the air pollution and living environment are getting worse and worse [1]. As a result, the air pollution has widened the gap between people's economic living conditions and quality of life. Since the atmospheric environment monitoring technology in China started late with the problem of slow development, the quantitative remote sensing monitoring of air pollution has become a hot spot [2]. The excessive pollution incidents that happened frequently in China has gradually received people's attentions to explore air pollution monitoring technology and awareness of protecting the environment. Therefore, improving air quality and protecting the environment are the urgent demand [3].
At present, the commonly used air pollution monitoring methods including satellite remote sensing monitoring, ground-based remote sensing monitoring, and manual instrument monitoring are currently used [4]. As for the manual instrument monitoring, it has the shortcomings of cumbersome operation and small monitoring area. The ground-based remote sensing monitoring is generally applied to monitor the local range, and it is only located in some regions. With the rapid development of aerospace technology, satellite remote sensing monitoring technology, as a new type of artificial intelligence technology, has been widely adopted in quantitative remote sensing monitoring of air pollution by virtue of its good real-time performance, strong periodicity, and wide coverage [5]. Considering the defects of the quantitative remote sensing monitoring algorithm of air pollution, such as low monitoring efficiency, small monitoring range, and low monitoring accuracy, it is 
necessary to study the quantitative remote sensing monitoring algorithm of air pollution.

According to the quantitative monitoring algorithm of air pollution based on geostationary satellite GOCI sensor proposed by Zhang et al., this algorithm, by combining with the theory of atmospheric aerosol change with time, the minimum fitting method, and time series iterative algorithm, mines the data of aerosol optical thickness and realizes the quantitative remote sensing monitoring of air pollution. However, in this algorithm, the monitoring time is long and there are many errors in the obtained monitoring results, so the monitoring efficiency and monitoring accuracy are low [6]. Zhang et al. proposed a quantitative remote sensing monitoring algorithm of air pollution based on MODIS data. By means of the $6 \mathrm{~S}$ model, this algorithm calculates the radiation transmission model equation under different aerosol optical thickness conditions, obtains the original spectral band information, and then acquires the surface reflectance information through pixel method [7]. In this way, it calculates the aerosol optical thickness so as to realize the remote sensing monitoring of air pollution. But this algorithm has the weaknesses of small monitoring coefficient as well as narrow monitoring range [8].

For the sake of solving the problems existing in the abovementioned algorithms, a quantitative monitoring algorithm of pollution based on artificial intelligence is proposed.

\section{Basic Theory of Atmospheric Radiation Transmission}

2.1. Equation of Atmospheric Radiation Transmission. In an uneven air medium, the interaction between light will affect the radiation intensity of light propagation [8].

Let $\lambda$ be the wavelength, $I_{\lambda}$ stand for the radiation intensity, and $I_{\lambda}+d I_{\lambda}$ stand for the radiation intensity corresponding to $I_{\lambda}$ after passing through the path in length ofds, then there is an equation as follows:

$$
\mathrm{d} I_{\lambda}=-k_{\lambda} \rho \times I_{\lambda} \mathrm{d} s,
$$

where $k_{\lambda}$ represents the mass extinction cross section corresponding to the radiation wavelength $\lambda$ and the transmission medium and $\rho$ represents the density corresponding to the transmission medium. As can be learned from above formula, the radiated electromagnetic wave will increase or decrease under the influence of different concentrations of aerosol [9]. The strength can be increased through the following equation:

$$
\mathrm{d} I_{\lambda}=j_{\lambda} \rho \mathrm{d} s,
$$

where $j_{\lambda}$ represents the source function coefficient and its physical meaning is similar to the mass extinction cross section; then, there is equation as follows:

$$
\mathrm{d} I_{\lambda}=-k_{\lambda} \rho \times I_{\lambda} \mathrm{d} s+j_{\lambda} \rho \mathrm{d} s .
$$

The above equation can be transformed into

$$
\frac{d I_{\lambda}}{k_{\lambda} \rho \mathrm{d} s}=-I_{\lambda}+J_{\lambda} .
$$

There is no particularity in the above radiation transmission equation, and all coordinate systems are available.

2.2. Beer-Bouguer-Lambert Law. Without regard to the influence of multiple scattering and emission on the radiation intensity during the research path and assume that the radiation transmission is a uniform medium, then there will be Beer-Bouguer-Lambert law [10].

Based on the Beer-Bouguer-Lambert law, the radiation transmission equation can be simplified as follows:

$$
\frac{\mathrm{d} I_{\lambda}}{k_{\lambda} \rho \mathrm{d} s}=-I_{\lambda}
$$

If the intensity $I_{\lambda}(0)$ is incident on $s=0$, the corresponding scattering intensity at $s_{1}$ can be obtained via the integration of above equation:

$$
I_{\lambda}\left(s_{1}\right)=I_{\lambda}(0) \exp \left(-\int_{0}^{s_{1}} k_{\lambda} \rho \mathrm{d} s\right) .
$$

Assume $k_{\lambda}$ does not depend on the distance $s$; that is, the medium extinction cross section is uniform and constant; let $u$ stand for the path length; then, the equation is as follows:

$$
u=\int_{0}^{s_{1}} \rho \mathrm{d} s .
$$

Then, there is the following equation:

$$
I_{\lambda}\left(s_{1}\right)=I_{\lambda}(0) \exp \left(-k_{\lambda} u\right) \text {. }
$$

Let $\tau_{\lambda}$ be the dielectric thickness of the medium existing between $s_{1}$ and $s_{2}$; then, its equation is as follows:

$$
\tau_{\lambda}=-\int_{s_{1}}^{s_{2}} k_{\lambda} \rho \mathrm{d} s^{\prime}
$$

2.3. Parallel Plane Atmospheric Radiation Theory. If the medium is divided, an infinite or several mutually parallel planes can be obtained, the light propagation planes are parallel, the internal properties of the layers in the plane are uniform, and the layers have different properties in the plane [11]. Let $z$ represent the linear distance corresponding to the vertical layered plane; then, the expression of the atmospheric radiation transmission equation without considering the monochromatic light wavelength variable is as follows:

$$
\cos \theta \frac{\mathrm{d} I(z, \theta, \phi)}{k \rho \mathrm{d} z}=-I(z, \theta, \phi)+J(z, \theta, \phi),
$$

where $\theta$ describes the angle of inclination with the upward perpendicular and $\phi$ describes the azimuth.

Let $\tau$ describe the vertical extinction thickness measured from the upper boundary of the atmosphere to the plane parallel atmosphere. When the entire extinction degree of the atmosphere can be expressed by $\tau, \tau$ becomes the optical thickness corresponding to the atmosphere. The equation is as follows: 


$$
\tau(z)=\int_{z}^{\infty} k \rho \mathrm{d} z^{\prime}
$$

In combination of equations (4) and (11), then

$$
\begin{array}{r}
\mu \frac{\mathrm{d} I(\tau, \theta, \phi)}{\mathrm{d} \tau}=I(\tau, \theta, \phi)-J(\tau, \theta, \phi), \\
\mu=\cos \theta .
\end{array}
$$

Without considering the infrared heat radiation problem, the scattering expression existing in the source function is the sum of multiple scattering and single scattering of solar radiation [12], and its calculation equation is as follows:

$$
J(\tau, \Omega)=\frac{\omega}{4 \pi} F_{0} e^{-e / \mu_{0}} p\left(\Omega, \Omega_{0}\right)+\frac{\omega}{4 \pi} \int_{4 \pi} I\left(\tau, \Omega^{\prime}\right) p\left(\Omega, \Omega^{\prime}\right) \mathrm{d} \Omega^{\prime},
$$

where the first item on the right describes the contribution of the single scattering of direct solar radiation in the direction $\Omega(\theta, \phi)$, the second item describes the contribution of the multiple scattering of the atmosphere, $\omega$ represents the reflection of the single scattering rate, $F_{0}$ represents the solar constant, $p$ represents the scattering phase function, and $\mu_{0}$ represents the cosine corresponding to the solar zenith angle.

By expanding the radiation transmission equation, the following formula is obtained:

$$
\begin{aligned}
\mu \frac{\mathrm{d} I(\tau, \theta, \phi)}{\mathrm{d} \tau}= & I(z, \theta, \phi)-\frac{\omega}{4 \pi} F_{0} e^{-e / \mu_{0}} p\left(\Omega, \Omega_{0}\right) \\
& -\frac{\omega}{4 \pi} \int_{4 \pi} I\left(\tau, \Omega^{\prime}\right) p\left(\Omega, \Omega^{\prime}\right) \mathrm{d} \Omega .
\end{aligned}
$$

2.4. Atmospheric Radiation Transmission Model. The positive and negative changes in solar radiant energy during atmospheric transmission can be expressed by processes such as atmospheric emission, absorption, and scattering [9]. The above processes usually produce a continuous spectrum, which is widely used in atmospheric environment monitoring based on the ability to avoid large quantitative errors appearing in the spectral inversion.

The expression formula of the $6 \mathrm{~S}$ atmospheric transmission model is as follows:

$$
\rho_{T}\left(\theta_{s}, \theta_{v}\right)=T_{g}\left(\theta_{s}, \theta_{v}\right)\left[\rho_{R+a}+T\left(\theta_{s}\right) T\left(\theta_{v}\right) \frac{\rho_{s}}{1-s \rho_{s}}\right] \text {, }
$$

wherein $\rho_{T}$ represents the total reflectivity corresponding to atmospheric transmission; $T_{g}\left(\theta_{s}, \theta_{v}\right)$ represents the transmittance, which is formed by atmospheric absorption; $\rho_{R+a}$ describes the reflectance of the radiation path formed by aerosol particles and molecular scattering; $T\left(\theta_{s}\right)$ represents the straight transmittance of the ground target and the solar path; $T\left(\theta_{v}\right)$ represents the linear transmittance of the sensor and the ground target path; $s$ represents the atmospheric hemispheric albedo; and $\rho_{s}$ represents the surface diffuse reflectance.
2.5. Electromagnetic Radiation Transmission Model. The complex process formed by the interaction between different media and electromagnetic waves is electromagnetic radiation transmission [13]. Solar radiations, such as radiation from the atmosphere and radiation from the ground, belong to the electromagnetic radiation received by the satellite sensor.

The radiance corresponding to the ground target recorded by a remote sensor is affected by the atmosphere. The scattering and absorption of the atmosphere will attenuate the electromagnetic radiation energy, while the path radiation of radiator can increase the electromagnetic radiation energy. Radiation transmission model generally describes the basic equation of electromagnetic radiation during atmospheric transmission. During atmospheric radiation transmission, the positive and negative changes in electromagnetic radiation energy can be expressed by the equation as follows.

(1) Atmospheric attenuation:

$$
\Delta I=-\rho \times k \times I \times \mathrm{d} s,
$$

where $I$ describes the emission energy and reflected energy corresponding to the ground object, that is, the incident brightness; $\Delta I$ describes the amount of energy change; $\rho$ describes the density corresponding to the scattering material or the absorbing material; $k$ is the sum of scattering coefficient and absorption coefficient, that is, the extinction coefficient; and $\mathrm{d} s$ describes the length of the optical path.

(2) Atmospheric heat radiation:

$$
\Delta I=\rho \times J \times \mathrm{d} s=\rho^{2} \times B(T) \times \mathrm{d} s,
$$

where $\rho$ describes the density corresponding to the absorbing material, $J$ describes the emission coefficient, $T$ is the thermodynamic temperature corresponding to the atmosphere, and $B$ is the Planck function.

(3) Atmospheric path radiation, which is equal to sky radiation:

$$
\Delta I=\omega_{0} \frac{K}{4 \pi} \rho^{2} \times \mathrm{d} s \int_{0}^{4 \pi} P\left(\Omega, \Omega^{\prime}\right) \times I\left(\Omega^{\prime}\right) \times \mathrm{d} \Omega^{\prime}-\rho \times k \times I,
$$

where $\omega_{0}$ describes a single scattering albedo, $P$ describes the scattering phase function of the scattering field angle distribution, and $\Omega$ describes the solid angle corresponding to the incident direction.

The influence of atmosphere on electromagnetic radiation includes refraction, absorption, reflection, and scattering. Due to the atmospheric influence, there is absorption effect existing in some bands of electromagnetic waves when the solar radiation passes through the atmosphere. Then, the 
radiation energy, which is affected by the absorption, will become the internal energy of the molecules, thereby causing the attenuation of solar radiation intensity in these bands.

Let $E_{0}$ represent the solar irradiance; the irradiance after atmospheric attenuation and by passing through the atmosphere path $x$ is $E^{*}$, and the formula is as follows:

$$
E^{*}=E_{0} e^{-k x}
$$

where $k$ describes the extinction coefficient, that is, the attenuation coefficient and $k x$ describes the optical thickness.

The electromagnetic wavelength and the atmospheric state determine the value of atmospheric attenuation. Let $L$ represent the total radiance obtained by the airborne remote sensor. The formula is as follows:

$$
L=L_{G} \tau+L_{P}
$$

where $\tau$ represents the atmospheric transmittance, $L_{P}$ represents the path radiation corresponding to the atmosphere, and $L_{G}$ represents the brightness corresponding to ground target after atmospheric attenuation; the calculation formula is as follows:

$$
L_{G}=\frac{E \rho}{\pi},
$$

where $E$ represents the irradiance corresponding to the ground target. On the basis of equation (21), the following formula is obtained:

$$
L_{G}=\frac{E \rho}{\pi}+L_{P}
$$

where parameter $E$ is related to atmospheric thickness and weather conditions; its calculation formula is as follows:

$$
E=\frac{E_{0}}{D^{2}} \times \cos (\theta) \times \tau,
$$

where $D$ represents the solar-ratio distance, which usually refers to the daily average distance; $E_{0}$ represents the solar constant; and $E_{0}$ represents the corresponding transmittance of the atmosphere.

\section{Quantitative Remote Sensing Monitoring Algorithm of Air Pollution}

In the near-infrared and visible-light bands, the pixel spectrum of satellite data includes two parts: surface reflected light and sky scattered light. Let $I$ represent the reflection spectrum received by the sensor; then, the expression formula is as follows:

$$
I=\left[I_{0}\left(T_{a} T_{m} T_{b}\right)^{u}+I_{m}+I_{b d}+I_{a d}\right] R_{g} T_{a} T_{m} T_{b}+I_{a}+I_{m}+I_{b},
$$

where $I_{0}$ represents the incident light intensity, $T_{a}$ represents the transmittance corresponding to anthropogenic aerosol, $T_{m}$ describes the transmittance of the atmospheric molecules, $T_{b}$ represents the transmittance of the background aerosol, $I_{m}$ represents the upward scattered light intensity of the atmospheric molecules, $I_{b d}$ represents the downward scattered light intensity corresponding to the background aerosol, $I_{a d}$ represents the downward scattered light intensity corresponding to the anthropogenic aerosol, $R_{g}$ represents the ground reflectivity, and $I_{a}$ represents the upward scattered light intensity corresponding to the anthropogenic aerosol.

Based on dividing by $I_{0}$ with the two sides of above equation, the following formula can be obtained:

$$
R_{g}=\frac{R-\omega_{a}-\omega_{m}-\omega_{b}}{\left[I_{0}\left(T_{a} T_{m} T_{b}\right)^{u}+\omega_{m}+\omega_{b d}+\omega_{a d}\right] T_{a} T_{m} T_{b}},
$$

where $R$ represents the pixel comprehensive reflectivity, $\omega_{a}$ represents the upward scattering rate corresponding to the anthropogenic aerosol, $\omega_{b}$ represents the upward scattering rate of the background aerosol, $\omega_{m}$ represents the upward scattering rate of the atmospheric molecule, $\omega_{b d}$ represents the downward scattering rate corresponding to the background aerosol, and $\omega_{a d}$ represents the downward scattering rate corresponding to the anthropogenic aerosol.

It can be deemed that the background aerosol is horizontally uniform within a scale of tens of miles, and the factors of season and geographical location will affect the type of background aerosol [14-17]. Based on the above analysis, the function of aerosol turbidity $\beta_{1}+\beta_{2}$ and $\beta_{3}$, as well as the pixel comprehensive reflectivity $R$ and the ground reflectivity, is obtained:

$$
R_{g}\left(R, \beta_{1}+\beta_{2}, \beta_{3}\right)=\frac{R-\omega_{a}-\omega_{m}-\omega_{b}}{\left[\left[I_{0}\left(T_{a} T_{m} T_{b}\right)^{u}+\omega_{m}+\omega_{b d}+\omega_{a d}\right] T_{a} T_{m} T_{b}\right]} .
$$

When the single leaf reflectance, water absorption rate, dry soil reflectance parameters, the direction of observation, and the direction of incidence are known, the pixel reflectance is a function of soil water content $V_{w s}$, vegetation leaf area index $L$, and wavelength. The expression formula is as follows:

$$
R_{g}\left(\lambda, V_{w s}, L\right)=R_{p}(\lambda, L)+R_{s}^{\prime}\left(\lambda, V_{w s}, L\right) .
$$

According to formula (27), the equation below is obtained:

$$
R_{g}\left(\lambda, V_{w s}, L\right)=R_{g}\left(R, \beta_{1}+\beta_{2}, \beta_{3}\right)
$$

By introducing the remote sensing data into the following equation, the only solution obtained is the aerosol turbidity:

$$
\left\{\begin{array}{l}
R_{g}\left(\lambda_{1}, \beta_{1+2}, \beta_{3}\right)=R_{g}\left(R_{1}, V_{w s}, L\right), \\
R_{g}\left(\lambda_{2}, \beta_{1+2}, \beta_{3}\right)=R_{g}\left(R_{2}, V_{w s}, L\right), \\
R_{g}\left(\lambda_{3}, \beta_{1+2}, \beta_{3}\right)=R_{g}\left(R_{3}, V_{w s}, L\right), \\
R_{g}\left(\lambda_{4}, \beta_{1+2}, \beta_{3}\right)=R_{g}\left(R_{4}, V_{w s}, L\right) .
\end{array}\right.
$$

The quantitative remote sensing monitoring of air pollution can be realized through the aerosol turbidity.

\section{Experiment and Analysis}

In order to verify the overall effectiveness of the artificial intelligence-based quantitative remote sensing monitoring 
TABLE 1: Monitoring time of three different algorithms.

\begin{tabular}{llll}
\hline DD & & JC & \\
& RG & JZ & SJ \\
\hline 1 & 0.2 & 0.6 & 1.1 \\
2 & 0.3 & 0.8 & 1.0 \\
3 & 0.2 & 0.7 & 1.2 \\
4 & 0.4 & 0.7 & 1.3 \\
5 & 0.3 & 0.8 & 1.3 \\
PJ & 0.28 & 0.72 & 1.18 \\
\hline
\end{tabular}

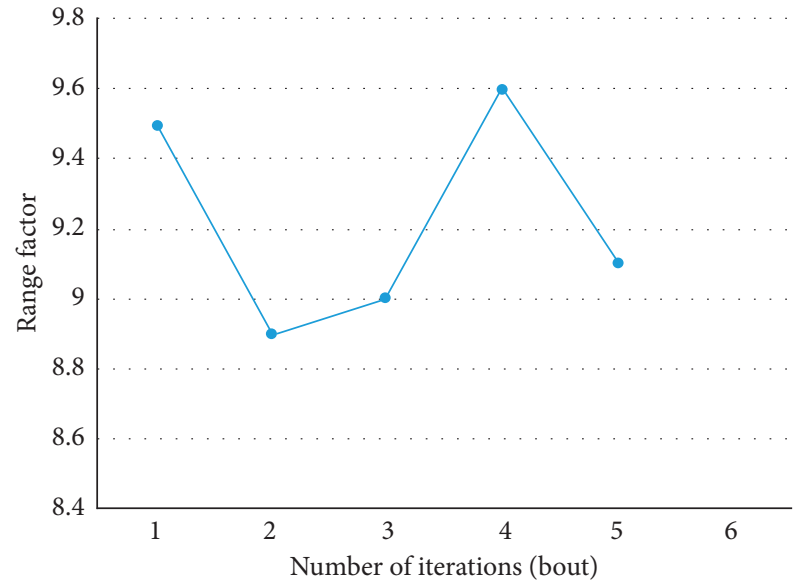

(a)

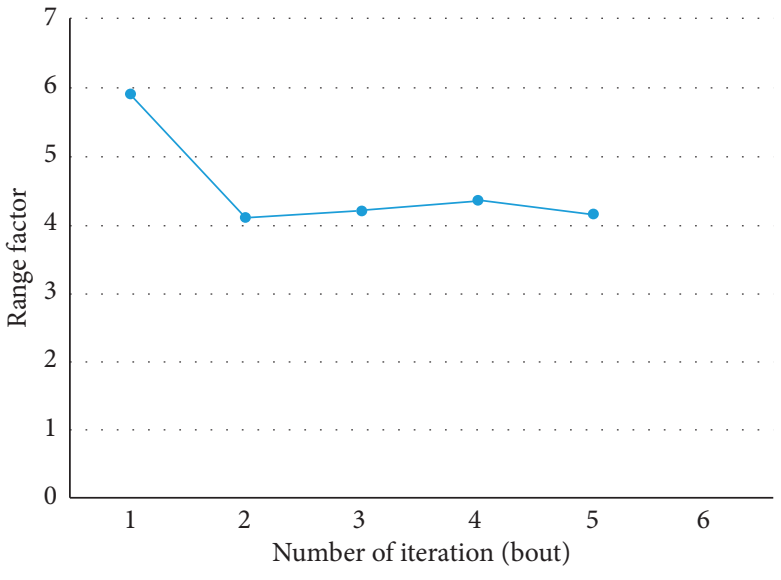

(b)

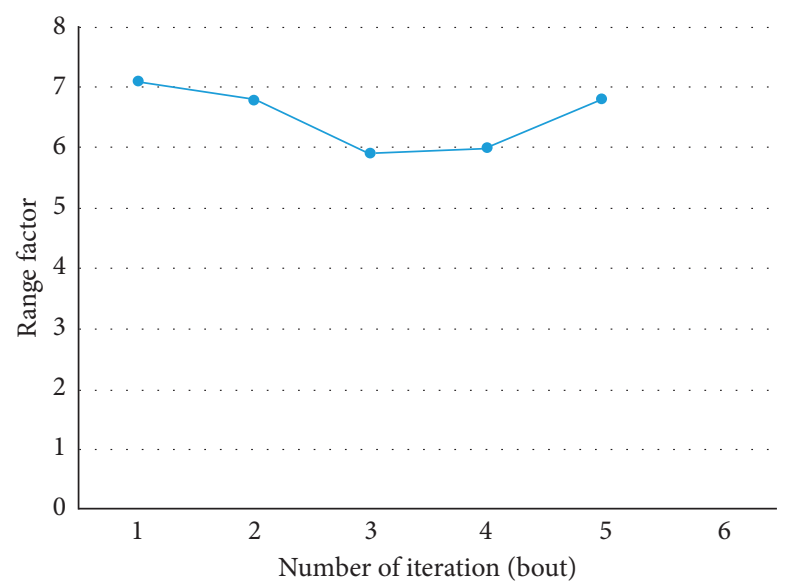

(c)

FIGURE 1: Range coefficient of (a) proposed algorithm, (b) algorithm based on geostationary satellite GOCI sensor, and (c) algorithm based on MODIS data.

algorithm of air pollution, a test is completed in the Linux platform. The monitoring times of three different algorithms of artificial intelligence-based quantitative remote sensing monitoring algorithm of air pollution, the geostationary satellite GOCI sensor-based quantitative remote sensing monitoring algorithm of air pollution, and MODIS data-based quantitative remote sensing monitoring algorithm of air pollution are compared. The test results are shown in Table 1.

In Table 1, DD represents the number of iterations of three different algorithms; JC represents the monitoring time of three algorithms, and the unit is s; RG represents the quantitative remote sensing monitoring algorithm of air pollution based on artificial intelligence; JZ represents the quantitative monitoring algorithm of air pollution based on geostationary satellite GOCI sensor; and SJ represents the quantitative remote sensing monitoring algorithm of air pollution based on MODIS data.

Analysis of the data in Table 1 shows that, in five iterations, the average monitoring time of the quantitative remote sensing monitoring algorithm of air pollution based on artificial intelligence is $0.28 \mathrm{~s}$; the quantitative monitoring algorithm of air pollution based on geostationary satellite GOCI sensor is $0.72 \mathrm{~s}$; and the quantitative remote sensing monitoring algorithm of air pollution based on MODIS data 


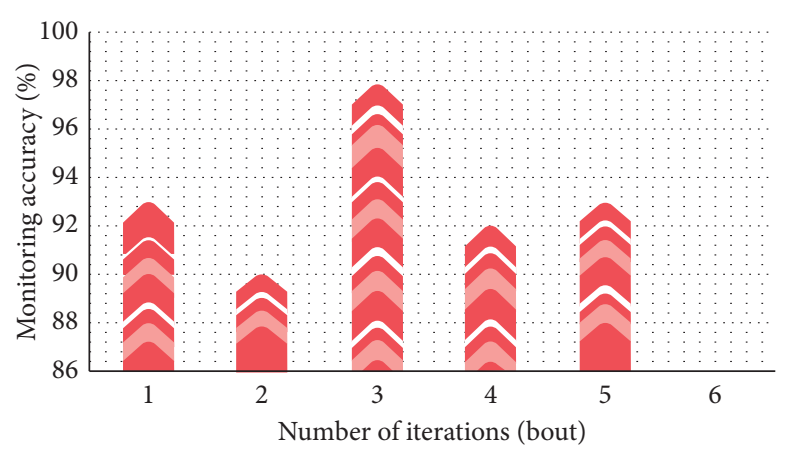

(a)

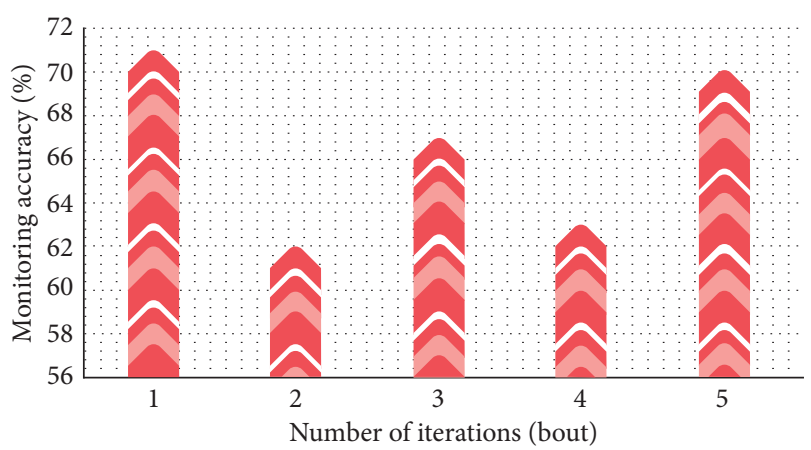

(b)

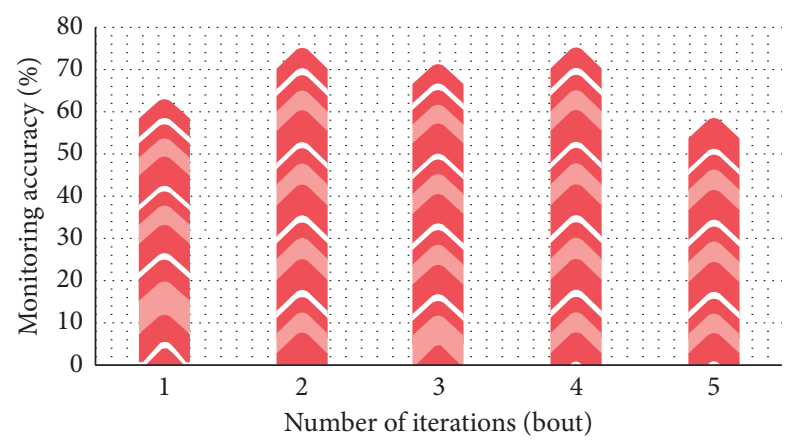

(c)

FIGURE 2: Monitoring accuracy of (a) proposed algorithm, (b) algorithm based on geostationary satellite GOCI, and (c) algorithm based on MODIS data.

is $1.18 \mathrm{~s}$. According to the comparison of the test results of three different algorithms, the quantitative remote sensing monitoring algorithm of air pollution based on artificial intelligence uses the shorted monitoring time. This is because the characteristics of atmospheric radiation transmission, Beer-Bouguer-Lambert law, parallel plane atmospheric radiation theory, and electromagnetic radiation transmission characteristics are analyzed before the quantitative monitoring, which have provided relevant information for quantitative remote sensing monitoring of air pollution, reduced the monitoring time, and improved the monitoring efficiency of quantitative remote sensing monitoring algorithm of air pollution based on artificial intelligence.

Let $\delta$ represent the range coefficient that takes value in the interval $[0,10]$. The larger the range coefficient, the larger the monitoring range of the algorithm. Based on taking range coefficient $\delta$ as a test index, the quantitative remote sensing monitoring algorithm of air pollution based on artificial intelligence, the quantitative monitoring algorithm of air pollution based on geostationary satellite GOCI sensor, and the quantitative remote sensing monitoring algorithm of air pollution based on MODIS data is tested. The results of comparing the monitoring scope of three algorithms are shown below.

Figure 1(a) is the test result of the quantitative remote sensing monitoring algorithm of air pollution based on artificial intelligence, which shows that the range coefficient of the quantitative remote sensing monitoring algorithm of air pollution based on artificial intelligence is above 8; Figure 1(b) is the test result of the quantitative monitoring algorithm of air pollution based on geostationary satellite GOCI sensor; Figure 1(c) is the test result of the quantitative remote sensing monitoring algorithm of air pollution based on MODIS data. The analysis of Figures 1(b) and 1(c) found that the range coefficients of the quantitative monitoring algorithm of air pollution based on geostationary satellite GOCI sensor and the quantitative remote sensing monitoring algorithm of air pollution based on MODIS data fluctuate around 5 and 7, respectively. As can be observed from comparing the results of the above algorithms, the range coefficient of the quantitative remote sensing monitoring algorithm of air pollution based on artificial intelligence is higher. Since the higher the range coefficient, the wider the monitoring range of the algorithm, and the monitoring range of the quantitative remote sensing monitoring algorithm of air pollution based on artificial intelligence is wider.

The quantitative remote sensing monitoring algorithm of air pollution based on artificial intelligence, the quantitative monitoring algorithm of air pollution based on geostationary satellite GOCI sensor, and the quantitative remote sensing monitoring algorithm of air pollution based on MODIS data are tested and compared in the monitoring accuracy. The results are shown below.

It can be seen from Figure 2 that, in multiple iterations, the monitoring accuracy of the quantitative remote sensing monitoring algorithm of air pollution based on artificial intelligence is higher than that of the quantitative monitoring algorithm of air pollution based on geostationary satellite GOCI sensor and the quantitative remote sensing 
monitoring algorithm of air pollution based on MODIS data. This is because the quantitative remote sensing monitoring algorithm of air pollution based on artificial intelligence constructs the pixel element combination simultaneous equation, which helps calculate the atmospheric aerosol turbidity and then realize the quantitative remote sensing monitoring of air pollution through the calculated aerosol turbidity. Therefore, this improved the monitoring accuracy of the quantitative remote sensing monitoring algorithm of air pollution based on artificial intelligence.

\section{Conclusions}

The rapid urbanization over the past years has increased energy consumption, worsened the air quality, and seriously threatened people's physical and mental health. Therefore, how to enhance air quality and protect the environment in fierce competition and development has become the main problem faced by the society. Urban environment is the result of urban construction and industrial production, and the urban air environment is receiving more attention since discovering its dangers. The premise of environmental governance and protection is environmental testing. However, the monitoring cost and station distribution will affect the routine monitoring of air pollution, making it difficult to obtain long-term and largescale air pollution information. On this consideration, the sensing data have been widely used in the field of air pollution monitoring because it is economical and macroscopic.

At present, the quantitative remote sensing monitoring algorithm of air pollution has the shortcomings of low monitoring efficiency, small monitoring range, and low monitoring accuracy. The proposed quantitative remote sensing monitoring algorithm based on artificial intelligence is proposed and can accurately detect a wide range of air pollution data within a short time, which has solved the problems existing in the current algorithm and laid a foundation for the development of quantitative remote sensing monitoring technology of air pollution.

\section{Data Availability}

We use simulation data, and our model and related hyperparameters are provided in our paper.

\section{Conflicts of Interest}

The authors declare that they have no conflicts of interest.

\section{Acknowledgments}

This work was supported by the Key Research Project of Natural Science in Anhui Province (KJ2019 A0681).

\section{References}

[1] W. Q. Liu, Z. Y. Chen, and J. G. Liu, "Air pollution optical remote sensing technology and development tendency," Environmental Monitoring in China, vol. 34, no. 2, pp. 1-9, 2018.
[2] Z. Yu, S. D. Liu, and Y. W. Wang, "Observation and analysis of variation characteristics of air pollutant concentration in urban area of Hangzhou city in 2014," Science Technology and Engineering, vol. 16, no. 16, pp. 95-104, 2016.

[3] N. Wang, T. R. He, and J. P. Liu, "Analysis on effect of summer precipitation in removing atmospheric pollutants in chongqing urban area," Environmental Engineering, vol. 35, no. 4, pp. 69-73, 2017.

[4] X. X. Zhang, Z. B. Yuan, and J. Y. Zhen, "Establishment of uncertainty evaluation method system for air pollutant monitoring data and analysis of its impact on PMF source analysis," Acta Scientiae Circumstantiate, vol. 39, no. 1, pp. 97-106, 2019.

[5] A. X. Cao, M. Ao, and L. C. Liang, "Mercury content in natural stone moss and biological indications of air pollution," Environmental Chemistry, vol. 35, no. 10, pp. 2204-2210, 2016.

[6] Y. H. Zhang, H. Q. Mao, and Q. Li, "Air pollution process AOD monitoring based on geostationary satellite GOCI sensor," China Environmental Science, vol. 38, no. 10, pp. 49-55, 2018.

[7] Z. H. Zhang, B. Mu, and H. You, "Application of PM2.5 retrieval in air pollution monitoring with MODIS data," Science of Surveying and Mapping, vol. 41, no. 9, pp. 42-46, 2016.

[8] F. Wang, Y. L. Han, and Y. Zhao, "Study on law of multi-time scale change of atmospheric particles PM10 and PM2.5 in Taiyuan city," Ecology and Environment, vol. 26, no. 9, pp. 1521-1528, 2017.

[9] F. Li, X. Tang, and Z. F. Wang, "Representative error estimation of ground observation of atmospheric pollutant concentration based on Beijing-Tianjin-Hebei high density ground observation network," Chinese Journal of Atmospheric Sciences, vol. 43, no. 2, pp. 277-284, 2019.

[10] L. Wang, B. L. Zi Bi, Y. Si Ma, and S. T. Yang, "Analysis of air pollution status in major cities in northern Xinjiang," Journal of Arid Land Resources and Environment, vol. 32, no. 6, pp. 182-186, 2018.

[11] M. H. Wang, W. C. Fu, and Z. P. Zhu, "Analysis of atmospheric visibility change characteristics in hangzhou from 1957 to 2016," Journal of Sichuan Agricultural University, vol. 35, no. 3, pp. 396-400, 2017.

[12] Z. M. Guo, Y. Liu, and X. F. Gao, "Absence from school in smog weather and analysis of influencing factors in chengdu primary school students," Chinese Journal of School Health, vol. 39, no. 11, pp. 84-87, 2018.

[13] Y. S. Lu, Y. X. Li, and B. Liu, "Hyper-spectral remote sensing smog monitoring based on deep residual network," Acta Optical Sinica, vol. 37, no. 11, pp. 314-324, 2017.

[14] J. B. Liu, J. Zhao, and Z. X. Zhu, "On the number of spanning trees and normalized Laplacian of linear octagonal-quadrilateral networks," International Journal of Quantum Chemistry, vol. 119, no. 17, Article ID e25971, 2019.

[15] J.-B. Liu, J. Zhao, and Z.-Q. Cai, "On the generalized adjacency, Laplacian and signless Laplacian spectra of the weighted edge corona networks," Physica A: Statistical Mechanics and Its Applications, vol. 540, p. 123073, 2020.

[16] J.-B. Liu, J. Zhao, H. He, and Z. Shao, "Valency-based topological descriptors and structural property of the generalized sierpiński networks," Journal of Statistical Physics, vol. 177, no. 6, pp. 1131-1147, 2019.

[17] J. L. Gu, H. S. Tang, and M. Liu, "Correlation analysis between mass concentration of atmospheric pollutants and aerosol optical thickness in Dalian," Scientia Geographical Sonica, vol. 39, no. 3, pp. 516-523, 2019. 